Citation: Rslan W. M. (2018) Sugar beet artificial seeds an overview. Highlights in BioScience, Volume 1.Article ID 20182, dio:10.36462/ H.BioSci.20182

Received: May 22, 2018

Accepted: July 17, 2018

Published: August 12, 2018

Copyright:@ $\odot 2018$ Rslan. This is an open access article distributed under the terms of the Creative Commons Attribution License, which permits unrestricted use, distribution, and reproduction in any medium, provided the original author and source are credited.

Data Availability Statement: All relevant data are within the paper

Funding: The authors have no support or funding to report.

\section{Date Palm Biotechnology: Recent Research an Overview}

\section{Wessam M. Rslan*}

Agricultural Genetic Engineering Research Institute; Agricultural Research Center, Egypt.

*To whom correspondence should be addressed: wessam.rslan@ageri.sci.eg

\section{Abstract}

Date palm (Phoenix dactylifera L.) is among the earliest fruit crops cultivated in the arid Arab Peninsula, North Africa, and Middle East territories. Dates are a significant source of food and revenue for Middle East and North Africa's local communities. It has distinctive features of biology and development that require special methods of reproduction, culture and governance. In varying dategrowing regions, there are thousands of date plant cultivars and varieties. The lengthy life cycle, long juvenile lifespan, and date palm dioecism produce cultivation difficult. Every year, the percentage of crop genomes sequenced has continued to increase. The incredible rate at which DNA samples become accessible is mainly due to the enhancement in cost-and speed-related sequencing techniques. Modern sequencing techniques enable the sequencing at realistic price of various cultivars of tiny plant genomes. Although many of the published genomes are deemed incomplete, they have nevertheless proven to be useful instruments for understanding significant plant characteristics such as fruit maturation, grain characteristics and adaptation of flowering time, here we review date palm genomic studies and determine its genomics element.

Keywords: Date palm, genome-wide association, SNP, genomics element, genome annotation . 


\section{Introduction}

Several of the plant genome sequences have been fully sequenced (collected, annotated and released) and have been openly accessible in the database, such as Oryza sativa (1), Zea mays (2), Hordeum vulgare (3), Phoenix dactylifera (4), Triticum aestivum (5), Eucalyptus grandis (6), Capsicum annuum (7), Beta vulgaris (8) and Solanum (9). Yang et al. (1) presented a full date palm chloroplast genome sequence of approximately $158,462 \mathrm{bp}$. They revealed that there are 112 distinctive genes and 19 duplicated parts in the IR areas in the date palm chloroplast genome. They recognized 78 SNPs within the population of a particular chloroplast genome as significant intravarietal polymorphisms, most of which were found in genes with essential tasks. They also discovered 18 polycistronic transcription blocks and 3 extremely expression-based genes-atpF, trnA-UGC, and rrn23 based on RNA-sequencing records.

Al-Dous et al. .(4) used the Illumina GAII sequencing platform to present the first date of the palm assembly draft genome sequence for the Khalas cultivar. They reported a date palm genome with size of $658 \mathrm{Mb}, 58 \%$ assembled sequences $(382 \mathrm{Mb})$ and forecast 25,059 genes. Among the nine cultivars of date palms trees (Deglet Noor, Deglet Noor BC5, Khalas, Khalas BC2, Medjool BC4, Medjool, Alrjeem, KhltMale, KhFxfem) they recorded more than 3.5 million polymorphic locations. In (http://qatar-weill.cornell.edu/ research/datepalmGenome/), this sequence was stored.

Fang et al. (12) provided date palm full sequence of mitochondrial genome with an estimated size of approximately 715.001 bp. Al-Mssallem et al. (13) introduced the second Khalas nuclear genome assembly, which is $605.4 \mathrm{Mb}$ in size, spanning $>90 \%$ of the genome $(\sim 671 \mathrm{Mb})$ and $>96 \%$ of its functional genes $(\sim 41,660$ genes). This sequence was stored with BioProject ID PRJNA83433 in Genbank (http://www.ncbi.nlm.nih.gov/) and produced the first genetic map of the date palm (Phoenix dactylifera) and recognized the putative gender chromosome. They submitted 4000 maps on the map using a total of 1293 cM of nearly 1200 molecular markers. These molecular markers were divided into 18 linkage clusters (LG) and proposed that the sex chromosome could be LG12.

Hazzouri et al. (14) launched an extensive catalog of about seven million single nucleotide polymorphisms in date palms based on a set of 62 cultivars re-sequencing the entire genome. Analysis of population structure suggested a significant genetic gap between North Africa and the date palms of Middle East/South Asia with proof of admixture in Egyptian and Sudanese cultivars. Candidate mutations for trait variation were recorded, including polymorphisms of nonsense and variety of presence/absence of gene material in important agronomic trait mechanisms. In the R2R3 myb-like orthologue of the oil palm virescens gene connected with fruit color variability, they also recognized a copy-like retrotransposon insertion polymorphism.

The first miRNA segments of the palm species Elaeis guineensis, Elaeis oleifera and Phoenix dactylifera were recorded by Silva et al. (15). In the genomes of these species, they recognized miRNA precursors and defined their potential biological functions proposed by the mature miRNA-based target gene regulation. Three hundred and thirty-eight precursors were recognized, varying in sequence size from 76 to 220 nucleotides dispersed in 33 households. In addition, they also recognized Musa acuminata 266 miRNA precursors that were phylogenetically near to palm species.

\section{Detection of resistance genes in date palm}

Al-Mssallem et al. (13) monitored Phoenix dactylifera protein samples using the Pfam NBS (NB-ARC) family PF00931 databases (E-value cutoff of 1.0) Hidden Markov Models (HMMs). Using an HMM model with Pfam TIR PF01582 (E-value cutoff 1.0) domains as well as LRR motifs in the C-terminal domains, the 144 expected amino acid sequences were used to identify TIR domains. In total, 144 non-redundant NBS-encoding proteins have been recognized and validated manually, accounting for about $0.35 \%$ of the gene designs in the Phoenix dactylifera genome.

\section{Simple sequence repeats in date palm}

Billotte et al. (16) built a library (GA) $n$ enriched with microsatellite and described 16 nuclear simple sequence repetition (SSR) loci in Phoenix dactylifera. They studied amplification and genotyping across taxes. This experiment reveals the usefulness of most SSR indicators in 11 other Phoenix organisms and the applicability of some of them in Elaeis guineensis, 11 Pritchardia species, Pritchardiopsis jeanneneyi species, and six Astrocaryum species.

Akkak et al. (17) separated from two microsatellite enriched date palm libraries forty-one easy sequence repeats (Phoenix dactylifera). Of the 41 SSRs, 17 chosen microsatellite loci were identified and assessed on a collection of 31 Algerian and Californian germplasm cultivars and clones. All primer pairs generated an amplification PCR pattern of the expected size and high polymorphism was detected among the samples analyzed. Tax amplification across the genus Phoenix indicates the usefulness of most SSR indicators in 14 other species.

Hamwieh et al. (18) researched the prevalence of microsatellite patterns in the first edition of the new genome profile of the date palm assembly produced by the next generation DNA sequencing of the entire genome shotgun. 
They structured a total of 1,091 primer combinations in simple/perfect microsatellite motifs flanking regions. From it 377 primers flanked dinucleotide, 352 primers flanked trinucleotide, and 362 primers pair flanked tetranucleotide repeats out of these primer combinations fifty primer pairs used for 8 Iraqi date palm varieties screening. Results showed that 28 combinations of primers were usable $(56 \%)$ and $18(36 \%)$ disclosed polymorphic alleles. Also (19) developed date palm molecular markers database.

Elmeer et al. (20) constructed thirty primer pairs of microsatellite genomic DNA markers for SSR. These molecular markers were used for eleven date palm genotypes to evaluate genetic diversity. Results stated that the targeted PCR fragments were not amplified by only seven (23.3 percent) of the thirty primers. While thirteen PCR primers (43.3\%) enhanced monomorphic banding patterns and polymorphic banding motifs were produced by the remaining ten primers $(33.4 \%)$.

Arabnezhad et al. (21) formed two SSR-enriched date palm genomic databases, including repeat motifs (AG) $n$ and (AAG) n, using 22 SSR PCR primer pairs to evaluate the genetic connection between 16 date palm cultivars cultivated in separate geographic areas (Iran, Iraq and Africa). Based on Nei's genetic distance they used, the cluster analysis put African date palms in a group other than the genotypes of Iran and Iraq. They stated it appears that African date palms ' domestication has taken a distinct path than those cultivated in the Middle East.

Bodian et al. (22) used easy sequence repeat markers to analyze genetic variation among 128 date palm specimens from the Figuig desert (Morocco). 121 females belonging to 11 cultivars were among those palms, and 7 were males. Eighteen SSR primers have been used, but all samples have been effectively amplified by only 15 primers. The dendrogram acquired showed three populations and male and female genotypes did not detect any genetic distinctions.

Zhao et al. (23) evaluated 28,889 samples of EST from the database of the date palm genome and recognized 4,609 ESTs as SSRs. Trinucleotide patterns $(69.7 \%)$ were the most common among these SSRs, followed by tetranucleotide $(10.4 \%)$ and dinucleotide patterns $(9.6 \%)$. They also engineered a total of 4,967 primer pairs from sequencing data for EST-SSR markers. Twenty of these primer pairs from twelve date palm cultivars were tested with genomic DNA. One-third of these primer pairs identified polymorphisms of DNA that distinguished the cultivars of the twelve date palms.

Aberlenc-Bertossi et al. (24) used the new sequence draft of the date palm genome Al-Dous et al. (11) to identify microsatellites and layout primers for SSR. They recognized 204 genes with microsatellite coding sequences, 150 of which were appropriate for primer construction, but only 103 had annealing locations that were not duplicated. With six or more repeats, such microsatellites had perfect trinucleotide motifs, and hexanucleotide motifs with at least four repeats. Of the 47 first pairs lastly maintained, 33 in a preliminary experiment with eight Phoenix dactylifera samples produced expected PCR amplification. The 33 loci have been further evaluated on 16 individuals comprising Phoenix roebelenii O'Brien(2), Phoenix dactylifera (7), Phoenix reclinata Jacq .(2), Phoenix rupicola T. Anderson (2), Phoenix theophrasti Greuter (2), and Phoenix canariensis interspecific hybrid phoenix sylvestris .

Zehdi-Azouzi et al. (25) used both nuclear SSR and chloroplast microsatellites to evaluate the variety and genome composition of date palm accessions from Mauritania to Pakistan in ten regions. A total of 295 accessions were evaluated using 18 nuclear SSR loci revealing > 200 alleles for every locus. They recorded analyzes of the date palm population's genetic composition using the Bayesian clustering strategy, hierarchical ranking of NJ and DACP. Their findings were obviously compatible with a two-cluster geographic structure. The first, called the Eastern pool, includes Djibouti, Iraq, Oman, Pakistan, and the UAE date palm accessions. While the Western pool was appointed the second cluster, it included the residual accessions from Africa, including Egypt, Algeria, Mauritania, Tunisia and Morocco (26).

\section{SNPs studies in date palm}

Al-Dous et al. (11) analyzed 3.5 million SNP across the genomes of male and female date palms trees to recognize gender-segregating polymorphisms. The findings noted best fit an XY sex-determination model with the heterogamous sex being males. Using a male heterogamete model 1,605 SNPs were noted segregating with gender. Analyzing two scaffolds with the most gender-segregating SNPs, they noted an estimated threefold distinction in divergence between male and female haplotypes from the reference sequence.

Across all genome sequences in these regions, the cultivars of 867 polymorphic sites have been observed. Comparing the females of Deglet Noor and Medjool with the female reference of Khalas disclosed that 253 and 271 sites varied from the reference of Khalas and that only 24 (9\%) and $19(7 \%)$ locations were heterozygous. At the same sites, their backcrossed males illustrated 736 and 770 different locations from the Khalas reference, of which $584(79 \%)$ and $578(75 \%)$ were heterozygous, respectively.

Al-Mssallem et al. (27) researched genetic variety of SNPs between many date tree species (Khalas, Fahal, Sukry, Agwa, Deglet Noor, Deglet Noor BC5, Medjool, Medjool BC4, KhalsFx, AlrijalF and Khalt) and recognized SNP 
(indel) abundances of $6.10(0.25), 5.51(0.15)$ and 6.24 (0.20) per kb. They explored how evolutionary selection left its marks in genomes of various date palm species by first assessing the allocation of SNPs on big scaffolds and then pinning down obviously chosen functional genes, particularly those strongly engaged in the metabolisms of sugar and energy. They found that there is no random distribution of SNPs, but a bimodal curve. Depending on SNPs and the frequency distributions, they built the phylogenetic tree of 11 Phoenix dactylifera cultivars.

In nine Saudi Arabian date plant cultivars (Sukkariat Al-Madinah Perny Al-Riyadh, Sukkariat Qassim, Rabia AlMadinah, Dekhaini Al-Riyadh, Ajwa Al-Madinah,, Shalaby Al-Madinah, Moshwaq Al-Riyadh and Moshwaq Hada AlSham), Sabir et al. (28) evaluated the entire maternal and plastid genome SNPs. They detected 188 SNPs in the mitochondrial genome at 37 distinct locations. For all nine cultivars, the amount of mutual SNPs was 16 . While there were 30 of the nine date palm cultivars in the amount of plastid SNPs, they were situated in 20 distinct genome locations with 13 in genes, 2 in introns, and 5 in intergenic spacers.

Hazzouri et al. (14) established an extensive catalog of about seven million SNPs in date palms depending on a set of 62 cultivars re-sequencing of the entire genome. Assessment of population structure indicates a significant genetic divide between North Africa and Middle East/South Asian date palms, with evidence of admixture in Egyptian and Sudanese cultivars. At least 56 genomic regions correlated with selective sweeps which may underlie regional adaptation were proposed by genome-wide selection scans. Candidate mutations for trait variety were recorded, including polymorphisms of nonsense and variety of presence/absence of gene material in important agronomic trait mechanisms. In the R2R3 myb-like orthologist of the oil palm virescens gene connected with fruit color variability, they also recognized a copy-like retro-transposon insertion polymorphism.

\section{Referances}

1. Yu J, Hu S, Wang J, Wong GK-S, Li S, Liu B, et al. A draft sequence of the rice genome (Oryza sativa L. ssp. indica). Science (80- ). 2002;296(5565):79-92.

2. Schnable PS, Ware D, Fulton RS, Stein JC, Wei F, Pasternak S, et al. The B73 maize genome: complexity, diversity, and dynamics. Science (80- ). 2009; 326 (5956): 1112-5.
3. International Barley Genome Sequencing Consortium. A physical, genetic and functional sequence assembly of the barley genome. Nature. 2012;491(7426):711-6.

4. Al-Dous EK, George B, Al-Mahmoud ME, Al-Jaber MY, Wang H, Salameh YM, et al. De novo genome sequencing and comparative genomics of date palm ( Phoenix dactylifera). Nat Biotechnol. 2011;29(6):521-7.

5. Peng Z, Lu Y, Li L, Zhao Q, Feng Q, Gao Z, et al. The draft genome of the fast-growing non-timber forest species moso bamboo (Phyllostachys heterocycla). Nat Genet. 2013;45(4):456-61.

6. Myburg AA, Grattapaglia D, Tuskan GA, Hellsten U, Hayes RD, Grimwood $J$, et al. The genome of Eucalyptus grandis. Nature. 2014;510(7505):356-62.

7. Qin C, Yu C, Shen Y, Fang X, Chen L, Min J, et al. Whole-genome sequencing of cultivated and wild peppers provides insights into Capsicum domestication and specialization. Proc Natl Acad Sci. 2014;111(14):5135-40.

8. Dohm JC, Minoche AE, Holtgräwe D, Capella-Gutiérrez $\mathrm{S}$, Zakrzewski F, Tafer $\mathrm{H}$, et al. The genome of the recently domesticated crop plant sugar beet (Beta vulgaris). Nature. 2014;505(7484):546-9.

9. Aversano R, Contaldi F, Ercolano MR, Grosso V, Iorizzo M, Tatino F, et al. The Solanum commersonii genome sequence provides insights into adaptation to stress conditions and genome evolution of wild potato relatives. Plant Cell. 2015;27(4):954-68.

10. Yang M, Zhang X, Liu G, Yin Y, Chen K, Yun Q, et al. The complete chloroplast genome sequence of date palm (Phoenix dactylifera L.). PLoS One. 2010;5(9):e12762.

12. Zhang T, Fang Y, Wang X, Deng X, Zhang X, Hu S, et al. The complete chloroplast and mitochondrial genome sequences of boea hygrometrica: Insights into the evolution of plant organellar genomes. PLoS One. $2012 ; 7(1)$

13. Al-Mssallem IS, Hu S, Zhang X, Lin Q, Liu W, Tan J, et al. Genome sequence of the date palm Phoenix dactylifera L. Nat Commun. 2013;4:2274.

14. Hazzouri KM, Flowers JM, Visser HJ, Khierallah HSM, Rosas U, Pham GM, et al. Whole genome re-sequencing 
of date palms yields insights into diversification of a fruit tree crop. Nat Commun. 2015;6:8824.

15. Da Silva AC, Grativol C, Thiebaut F, Hemerly AS, Ferreira PCG. Computational identification and comparative analysis of miRNA precursors in three palm species. Planta. 2016;243(5):1265-77.

16. Zehdi S, Trifi M, Billotte N, Marrakchi M, Pintaud JC. Genetic diversity of Tunisian date palm (Phoenix dactylifera) revealed by nuclear microsatellite polymorphism. Hereditas. 2004;141:278-87.

17. Akkak A, Scariot V, Torello Marinoni D, Boccacci P, Beltramo C, Botta R. Development and evaluation of microsatellite markers in Phoenix dactylifera L. and their transferability to other Phoenix species. Biol Plant. 2009;53(1):164-6.

18. Hamwieh A, Farah J, Moussally S, Al-Shamaa K, Almer $\mathrm{K}$, Khierallah $\mathrm{H}$, et al. Development of 1000 microsatellite markers across the date palm (Phoenix dactylifera L.) genome. Acta Hortic. 2010;882:269-77.

19. Mokhtar MM, Adawy SS, El-Assal SE-DS, Hussein EHA. Genic and Intergenic SSR Database Generation, SNPs Determination and Pathway Annotations, in Date Palm (Phoenix dactylifera L.). PLoS One. Public Library of Science; 2016;11(7):e0159268.

20. Elmeer K, Sarwath H, Malek J, Baum M, Hamwieh A. New microsatellite markers for assessment of genetic diversity in date palm (Phoenix dactylifera L.). 3 Biotech. 2011;1:91-7.

21. Arabnezhad H, Bahar M, Mohammadi HR, Latifian M. Development, characterization and use of microsatellite markers for germplasm analysis in date palm (Phoenix dactylifera L.).Sci Hortic (Amsterdam).2012;134:150-6.

22. Bodian A, Elhoumaizi MA, Ndir NK, Hasnaoui A, Nachtigall M, Wehling P. Genetic diversity analysis of date palm (Phoenix dactylifera L.) cultivars from Figuig oasis (Morocco) using SSR markers. Int J Sci Adv Technol. 2012;2(3):96-104.
23. Zhao Y, Williams R, Prakash CS, He G. Identification and characterization of gene-based SSR markers in date palm (Phoenix dactylifera L.). BMC Plant Biol. 2013;12:237.

24. Aberlenc-Bertossi F, Castillo K, Tranchant-Dubreuil C, Chérif E, Ballardini M, Abdoulkader S, et al. In silico mining of microsatellites in coding sequences of the date palm (Arecaceae) genome, characterization, and transferability. Appl Plant Sci. 2014;2(1):1300058.

25. Zehdi-Azouzi S, Cherif E, Moussouni S, GrosBalthazard M, Naqvi SA, Lude??a B, et al. Genetic structure of the date palm (Phoenix dactylifera) in the Old World reveals a strong differentiation between eastern and western populations. Ann Bot. 2015;116:101-12.

26. Adawy SS, Mokhtar MM, Alsamman AM, Sakr MM. Development of annotated EST-SSR database in olive (Olea europaea). Int J Sci Res. 2015;4(9):1063-73.

27. Al-mssallem IS, Hu S, Zhang X, Lin Q, Liu W, Tan J, et al. Genome sequence of the date palm Phoenix. Nat Commun. Nature Publishing Group; 2013;4:1-9.

28. Sabir JSM, Arasappan D, Bahieldin A, Abo-Aba S, Bafeel S, Zari TA, et al. Whole mitochondrial and plastid genome SNP analysis of nine date palm cultivars reveals plastid heteroplasmy and close phylogenetic relationships among cultivars. PLoS One. 2014;9(4): e94158. 